\title{
ILCEA
}

Revue de l'Institut des langues et cultures

d'Europe, Amérique, Afrique, Asie et Australie

16 | 2012

La culture progressiste à l'époque de la guerre froide

\section{Notices bio-bibliographiques}

\section{OpenEdition \\ Journals}

Édition électronique

URL : http://journals.openedition.org/ilcea/1595

DOI : 10.4000/ilcea.1595

ISSN : 2101-0609

\section{Éditeur}

UGA Éditions/Université Grenoble Alpes

\section{Édition imprimée}

ISBN : 978-2-84310-232-5

ISSN : $1639-6073$

\section{Référence électronique}

" Notices bio-bibliographiques », ILCEA [En ligne], 16 | 2012, mis en ligne le 04 juillet 2012, consulté le 22 mars 2021. URL : http://journals.openedition.org/ilcea/1595 ; DOI : https://doi.org/10.4000/ilcea. 1595

Ce document a été généré automatiquement le 22 mars 2021.

(C) ILCEA 


\section{Notices bio-bibliographiques}

1 Michèle ALTEN. MCF à l'université Paris-Sorbonne. Thèse en histoire contemporaine, Habilitation en musicologie. Publications sur l'histoire de l'enseignement musical et sur l'histoire de la démocratisation de la musique (recensées sur le site du groupe de recherche Patrimoines et langages musicaux).

Courriel : <michele.alten@paris-sorbonne.fr>

2 Tatjana BÖHME-MEHNER. Université de Halle-Wittenberg, étudie la musicologie et le journalisme à l'université de Leipzig, soutient son doctorat en musicologie en 2003. Enseigne depuis 1998 dans les universités de Leipzig, Halle, Weimar/Jena (Allemagne). Ses publications couvrent un large spectre de sujets. Depuis 2003, elle se spécialise dans le domaine de la musique électroacoustique pour obtenir l'habilitation à diriger des recherches. Chercheur associé au MINT-OMF (Paris 4 -Sorbonne), à l'Ina-GRM et membre du CIERA (EHESS), du DEGEM depuis 2003 et de l'EMS depuis 2006.

Courriel : <Tatjana.Mehner@t-online.de>

3 Noemi DE HARO GARCíA. Enseignante et chercheuse docteur avec un contrat « Juan de la Cierva » au département d'Histoire et Théorie de l'Art de l'Universidad Autónoma de Madrid. Publications sur l'histoire de l'art espagnol contemporain, les relations entre culture visuelle et politique et la méthodologie de l'histoire de l'art. Son dernier livre paru est Grabadores contra el franquismo (Madrid, 2010). À présent elle développe un projet de recherche postdoctoral sur les identités dissidentes tolérées pendant la transition démocratique en Espagne.

Courriel : <noemi_deharo@yahoo.es>

4 Isabelle DESPRÉS. Ancienne élève de l'ENS de Fontenay, agrégée de russe, professeur de langue et littérature russes et directrice du Centre d'études slaves contemporaines de l'université Stendhal, à Grenoble, Isabelle Després est l'auteur d'une thèse sur la critique littéraire dans les revues moscovites de l'époque romantique. Elle consacre actuellement ses travaux de recherche à la littérature, la poésie et la critique littéraire russe contemporaine.

Publications en rapport avec le sujet proposé :

Isabelle Després, «Le discours nationaliste chez les critiques religieux en Russie contemporaine ", dans M. Niqueux (dir.), Religion et Nation (actes de la journée d'étude 
organisée par l'Institut européen Est-Ouest (UMR 5206), ENS Lyon, le 8 juin 2009) [en ligne], Lyon, ENS LSH, mis en ligne le 23 juillet 2010. Disponible en texte intégral sur $<$ http://institut-est-ouest.ens-lyon.fr/spip.php?rubrique95>

-, «Les paraphrases de Timour Kibirov », Chroniques Slaves, $\mathrm{n}^{\circ} 4$, Grenoble, 2008.

-, «La réception et la transposition des concepts occidentaux en Russie. Postmodernisme et post-modernité », dans S. Martin (dir.), Circulation des concepts entre Occident et Russie [en ligne], Lyon, ENS LSH, mis en ligne le 10 décembre 2008. Disponible sur <http://institut-est-ouest.ens-lsh.fr/spip.php?article>

-, «Modernisme et postmodernisme: une révision des valeurs de l'Âge d'argent à l'époque de la post modernité ?", L'Âge d'Argent dans la culture russe, Modernités russes, Lyon, 2008, p. 659-670.

-, « Le Mot dans la nouvelle prose russe, l'exemple du Slynx de Tatiana Tolstaïa », La revue russe, Paris, $\mathrm{n}^{\circ}$ 21, 2002, p. 51-63.

-, "Le postmodernisme a-t-il des perspectives? Littérature russe contemporaine ", dans Essais sur le discours de l'Europe éclatée, Grenoble.

Courriel : <Isabelle.Despres@u-grenoble3.fr>

5 Jürgen DOLL. Professeur d'études germaniques à l'université Paris Est Créteil (UPEC). Il a fait ses études de germanistique et romanistique à Vienne, puis un doctorat et une HDR en France. Son doctorat en Autriche portait sur le dramaturge espagnol ValleInclán, son doctorat en France sur l'auteur autrichien Jura Soyfer (mort à Buchenwald), son habilitation sur le théatre politique en Autriche entre les deux guerres (Theater im Roten Wien, Vienne-Cologne-Weimar, Böhlau, 1997, 454 p.) De nombreuses publications sur l'austromarxisme, le théâtre politique et populaire, K. E. Franzos, J. Soyfer, E. Fried, $\mathrm{J}$. Améry, la littérature et la civilisation allemandes et autrichiennes des $\mathrm{XIX}^{\mathrm{e}}$ et $\mathrm{xx} \mathrm{x}^{\mathrm{e}}$ siècles.

Derniers livres parus :

Jürgen Doll (dir.), Jean Améry. De l'expérience des camps à l'écriture engagée, Paris, l'Harmattan, 2006.

-, Exil antinazi, témoignages concentrationnaires, Paris, Éditions Indigo, 2008.

Courriel: <jurgen.doll@wanadoo.fr>

6 François GENTON. Né en 1955, études à Nancy, ENS de Saint-Cloud, Paris 10-Nanterre et Ratisbonne. Professeur à l'université Stendhal-Grenoble 3. Publications sur l'histoire de la littérature, des idées, de la chanson et du cinéma en Allemagne.

Dernier livre paru :

Denis Bonnecase et François Genton (dir.), Ferments d'ailleurs. Transfert culturels entre Lumières et romantisme, Grenoble, ELLUG, 2010, 370 p.

Site professionnel (avec liste des publications): <http://w3.u-grenoble3.fr/ilcea/ ceraac/spip.php?breve4>

Courriel : <francois.genton@u-grenoble3.fr>

7 Eckhart GILLEN. Historien de l'art, critique d'art, nombreux articles et nombreux livres sur l'art dans les deux Allemagnes. Curateur et initiateur de plusieurs expositions et éditeur de leurs catalogues, par exemple de l'exposition R. B. Kitaj. Eine Retrospektive (Berlin, Musée juif, 27 septembre 2012-21 janvier 2013).

Dernier livre paru :

Eckhart Gillen, Feindliche Brüder. Der Kalte Krieg und die deutsche Kunst 1945 bis 1990, Berlin, 2009.

Courriel : <e.gillen@t-online.de> 
Stéphanie GONÇALVES. Assistante et doctorante en histoire contemporaine à l'Université Libre de Bruxelles, au département des Sciences de l'Information et de la Communication. Son sujet de thèse porte sur les tournées de ballet à Paris et à Londres pendant la guerre froide (1947-1968), sous la direction d'Irene Di Jorio.

Courriel : <sgoncalv@ulb.ac.be>

9 Laurence GUILLON (DUCHAINE). Maître de conférences en civilisation allemande à l'université de Paris-Ouest (Nanterre). Ancienne élève de l'ENS Lyon, agrégée d'allemand, enseignante dans le supérieur depuis 2006 (AMN durant 3 ans à Paris 3, ATER pendant un an à Paris 4). Thèse intitulée: "(Re)construire dans la division. Aspects de la vie juive à Berlin entre Est et Ouest (1945-1990)", soutenue en 2009 à l'université de la Sorbonne-Nouvelle Paris 3 et publiée en 2012 à Paris, Éditions CNRS : La vie juive à Berlin après 1945.

Courriel : <laurenceguillon@yahoo.fr>

Barbara et Winfried JUNGE. Né en 1935 à Berlin, Winfried Junge a perdu ses parents à un jeune âge. Il entame en 1954 des études cinématographiques à Potsdam-Babelsberg (Babelsberg) en 1954, débute comme critique de cinéma et rejoint en 1961 les studios de la DEFA (RDA) pour le cinéma documentaire. Il est l'auteur de plus de 50 films documentaires, dont 19 ont été tournés pour Les Enfants de Golzow (1961-2011), le premier et plus long projet d'observation de longue durée de l'histoire du cinéma. Au cours de sa carrière, Winfried Junge et ses films ont reçu de nombreuses récompenses, en Allemagne et à l'étranger. Barbara Junge, née en 1943, traductrice-interprète de formation, a d'abord supervisé le doublage des films de la DEFA en langue étrangère (rédaction et direction des comédiens). Elle rejoint le projet Les Enfants de Golzow en 1978, monte tous les films liés à ce projet depuis 1983 et signe avec Winfried Junge la réalisation depuis 1992. Voir le site «Lebensläufe. Die Kinder von Golzow » : <http:// www.kinder-von-golzow.de/>

Courriel : <info@kinder-von-golzow.de>

11 Elise LANOË. Née en 1981, ancienne élève de l'ENS Lyon, agrégée d'allemand, ATER à l'université Lille 3 et titulaire d'un doctorat en Études germaniques soutenu en mai 2012 à l'université Lille 3. Sujet de la thèse : « Les politiques culturelles extérieures de la RFA et de la France au Brésil (1961-1973) ». Déjà auteur de comptes rendus et de divers articles sur son sujet de thèse, voire sur les «Problèmes méthodologiques de l'interdisciplinarité dans la recherche en civilisation allemande ».

Courriel : <elise.lanoe@free.fr>

12 Claudia MAREIS. Spécialiste de l'histoire du design et des arts appliqués. A soutenu en 2010 une thèse des universités des arts de Linz (Autriche) et Zurich sur le sujet du design en tant que culture épistémique. Les interférences entre les discours sur le design et sur la connaissance depuis 1960. Thèse publiée en 2011. A travaillé jusqu'en 2009 comme chercheuse sur la théorie du design à Berne. Depuis 2011 à l'université de Bâle où elle travaille sur La découverte systématique durant le court $\mathrm{XX}^{e}$ siècle. Étude de cas sur l'histoire et la pratique de la créativité et des techniques d'idéation. Membre de plusieurs sociétés savantes allemandes, suisses et internationales sur la théorie du design et la recherche dans ce même domaine. Nombreuses publications et éditions à côté de la thèse.

Courriel : <claudia.mareis@unibas.ch> 
Stefan MAURER. Doctorant, chercheur à l'université de Vienne, dans le cadre du projet : « Discours de la guerre froide ». Déjà plusieurs publications, éditeur de l'auteur et journaliste Rudolf Kalmar (2009), articles sur Vienne après 1945.

Courriel : <stefan.maurer@univie.ac.at>

Patrice NEAU. Né en 1954, Professeur au département d'Études germaniques de l'université de Nantes, spécialiste de civilisation des pays de langues allemande. Mes recherches portent (ou ont porté) sur différentes thématiques :

- l'Allemagne de Guillaume II ;

- les minorités germanophones en Transylvanie et dans le Banat ;

- les arts plastiques en RDA.

Derniers articles :

Patrice Neau, «Vom Umgang mit den ,Parteiorganen der Kulturpolitik ${ }^{\star}$ der DDR. Strategien, um die Zensur zu unterlaufen am Beispiel der Leipziger Schule ", dans Die Stärke der Schwäche, Schriften des Collegium Pontes, Görlitz, Peter Lang, 2009, p. 29-46.

-, « „Die Kluft zwischen Kunst und Volk überwinden“. Ideologische Kämpfe in den bildenden Künsten der SBZ und der DDR am Beispiel der ersten drei Deutschen Kunstausstellungen (1946-1953)», Congrès de l'AGES, Göttingen, 2008 (en cours de publication).

-, « „60 Jahre, 60 Werke“. Une autocélébration de la République de Bonn », Allemagne d'aujourd'hui, $\mathrm{n}^{\circ} 189$, juillet-septembre 2009, p. 302-312.

-, «La représentation de la famille dans la peinture de la RDA », La famille de/en RDA (journée d'études du Centre Inter-Langues, Textes/Images/Langages, 15 mai 2009, université de Bourgogne, Dijon) (en cours de publication).

-, «Renaissance d'une culture ouvrière ou imposition d'un modèle idéologique: artistes peintres et peintres amateurs dans l'Allemagne de l'Est (1945-1959) » (Congrès de l'Âges, université de Lille III - Charles de Gaulle, mai 2010) (en cours de publication). Courriel : <Patrice.neau@univ-nantes.fr>

15 Doris NEUMANN-RIESER. Chercheur à l'université de Vienne, dans le cadre du projet : « Discours de la guerre froide ». Plusieurs conférences et publications, thèse en cours sur le réalisme de Brecht.

Courriel : <doris.neumann-rieser@univie.ac.at>

Paloma OTAOLA GONZÁLEZ. Professeur des universités IETT (Institut d'Études Transtextuelles et Transculturelles), Faculté des Langues à l'université Jean Moulin Lyon 3. Projet de recherche en cours sur la musique pop dans l'Espagne franquiste.

Courriel : <paloma.otaola@univ-lyon3.fr>

17 Edmond RAILLARD. Professeur à l'université Stendhal-Grenoble 3, membre de l'ILCEA, traducteur littéraire (fiction, esthétique, écrits de peintres). Ancien membre de l'École des hautes études hispaniques (Casa de Velázquez). Travaille sur la littérature catalane contemporaine, les arts plastiques et le cinéma dans le monde hispanique.

Page personnelle : <http://w3.u-grenoble3.fr/ilcea/cerhius/spip.php?breve7>

Courriel : <edmond.raillard@u-grenoble3.fr>

18 Kathrin RAMINGER. Mag. Kathrin Elisa Raminger. Études ibériques et d'archéologie à Vienne. Depuis 2009 doctorante sur Expositions et politique durant les ères Franco et Salazar. A publié un article sur ce sujet. Nombreux stages (fouilles archéologiques, Espagne, 
Turquie, etc.; membre de l'équipe coordonnant un festival de danse en 2007 France Danse Europe/Autriche : Nouvelle Vague).

Courriel : <kathrin.raminger@univie.ac.at>

Andreas RAUCH. Depuis 2011 enseignant à l'université de Chemnitz; 2010, ATER d'allemand à l'université de Reims / CUT de Troyes ; 2004-2010, lecteur d'allemand de l'office Allemand d'Échanges Universitaires (DAAD) à l'université de Paris 10 Nanterre, doctorant en Études germaniques à l'université de Lyon 2, Études romanes et anglo-américaines, Science de l'éducation, Psychologie à Zwickau et Chemnitz (Allemagne) ; 1994-1995, assistant d'allemand au Lycée "Pierre Forest » à Maubeuge (Nord) ; 2001, premier examen d'État : mémoire sur l'utilisation des chansons en cours d'anglais sous l'angle particulier des composantes motivationnelles et didactiques, professeur stagiaire au Lycée « Pufendorf » à Flöha (Allemagne); 2004, second examen d'État («Possibilités d'intégration des chansons dans les cours didactiques»); 2004, professeur à l'École des langues étrangères appliquées BFS à Chemnitz; 2006, inscription en première année de thèse à l'université de Lyon 2 , directeur de thèse : Jacques Poumet. Sujet de thèse : Tendances et évolution de la chanson en RDA.

Publications récentes :

Andreas Rauch, "Dr. Blues: Theo Lehmann, un pasteur contestataire", Le nouveau bulletin de l'A.D.E.A.F., $\mathrm{n}^{\circ}$ 105, Paris, septembre 2009, p. 19-23.

-, La République démocratique allemande dos au mur (université de Paris 8, 2009), Conception, traduction de textes et choix des chansons est-allemandes. Exposition en collaboration avec Jean Mortier et Mathilde Roussat.

Courriel : <rauchand@gmail.com>

20 Marta RUIZ. Maître de conférences à l'université Stendhal - Grenoble 3. A soutenu en 2001 une thèse explorant les rapports entre l'engagement politique et l'œuvre autobiographique et narrative de Jorge Semprun (Jorge Semprun: réécriture et mémoire idéologique). Depuis, elle participe aux travaux de l'ILCEA-CERHIUS et continue à s'intéresser à l'histoire intellectuelle d'un point de vue comparatif en explorant les rapports entre le politique et les productions culturelles au sens large au $\mathrm{xx}^{\mathrm{e}}$ siècle.

Elle a notamment publié :

Marta Ruiz, «Cuadernos del Congreso por la Libertad de la Cultura: anticomunismo y guerra fría en América Latina », El Argonauta español, nº 3, janvier 2006. Disponible sur <http://argonauta.imageson.org/document75.html>

-, « ¿Vendidos al enemigo?”: Disidentes y renegados del comunismo español (1921-1956)» [en ligne], Nuevo Mundo, Mundos Nuevos, Coloquios, 2009. Disponible sur $<\mathrm{http}$ ///nuevomundo.revues.org/index56330.html>

-, "Jorge Semprun : autobiographie, réécriture et vérité », Les cahiers de l'ILCEA, $\mathrm{n}^{\circ} 7$, 2004/2005 : « Littérature et vérité », Grenoble, ELLUG, 2005, p. 177-192.

Courriel : <marta.ruiz-galbete@u-grenoble3.fr>

Jacek RZESZOTNIK. Prof. Dr. habil., Germanist, Prodekan der Philologischen Fakultät der Universität Wroclaw/Breslau (Polen) und Leiter der Forschungsstelle für Literatur und Medien am Germanistischen Institut der Universität Wroclaw/Breslau. Forschungsschwerpunkte: Literaturwissenschaft (deutsch-, englisch- und polnischsprachige Unterhaltungsliteratur im 19. und 20./21. Jh.; literarische Kommunikationsstrategien; Rezeption spekulativer Literatur in Polen und im deutschen Sprachraum; Rezeption von Stanislaw Lem im deutschen Sprachraum) und 
Medienwissenschaft (intermediale ästhetische Transformationsprozesse; Neue Medien; Internetliteratur). Mitbegründer und Mitherausgeber des wissenschaftlichakademischen Periodikums „Zeitschrift für Fantastikforschung“ (Universität Hamburg). Rund 180 Veröffentlichungen (Monografien, Herausgeberschaften, Texteditionen, Aufsätze, Enzyklopädie-Einträge etc.).

Courriel : <jacekrz@uni.wroc.pl>

Václav ŠMIDRKAL. Doktorand im Fach Moderne Geschichte an der Karls-Universität Prag. Forschungsschwerpunkt: Kultur und Staat in sozialistischen Ländern Ostmitteleuropas.

Courriel : <smidrkalv@centrum.cz>

Günther STOCKER. Professeur à l'université de Vienne. Études germanistique et de journalisme à Salzbourg et Zurich. Sa recherche porte sur les relations entre médias et littérature, l'intermédialité, la littérature d'après 1945, de la guerre froide. Très nombreuses publications. Les plus récentes porte sur la littérature autrichienne des années 1950 et de l'époque de la guerre froide. Il dirige le projet « discours de la guerre froide", un site très riche, disponible sur <http://germanistik.univie.ac.at/kkdiskurse>

Courriel : <guenther.stocker@univie.ac.at>

David TUAILLON. Docteur en arts du spectacle de l'université Bordeaux 3 - Montaigne. Il a soutenu en 2009 une thèse consacrée aux Pièces de guerres d'Edward Bond dont il est l'un des spécialistes de l'œuvre en France. Il a ainsi publié plusieurs articles et ouvrages de vulgarisation sur cet auteur, dont la seule monographie multimédia qui lui est dédiée en langue française (numéro 1 de la revue électronique Colline.fr), traduit plusieurs de ses écrits théoriques (en particulier pour la collection Lexi/Textes du Théâtre national de la Colline ou dans la revue Théâtre/Public)et achève actuellement un livre d'entretiens avec lui à paraître aux éditions Les Belles Lettres. Depuis 2002, il a été chargé d'enseignement dans les universités Paris 1 - Panthéon-Sorbonne, Paris 3 Sorbonne-Nouvelle, Lille 3 - Charles de Gaulle. Il a commis des articles dans les revues Europe, Théâtre-Public, Comédie Française - Les Cahiers, Les Inrockuptibles, Hystrio ou Alphabeta 2. Entre 2000 et 2009, il a régulièrement travaillé au Théâtre national de la Colline où il a été l'un des collaborateurs directs d'Alain Françon. En 2009, il a réalisé avec lui le livre retraçant ses années à la direction de ce théâtre: Quittez le théâtre affamés de changements, Biro éditeur.

Courriel : <davidtuaillon@sfr.fr>

Nadine WILLMANN. Née en 1961. PRAG en études allemandes à l'Institut d'études politiques à l'université de Strasbourg. Doctorat en études germaniques (direction: Prof. Francois Genton) (« Günther Weisenborn (1902-1969), un écrivain de la résistance allemande »), Études à l'ENS Saint-Cloud et à Paris 10.

Dernières publications :

Nadine Willman, Günther Weisenborn, un écrivain de la résistance allemande, Paris, L 'Harmattan, 2007, 354 p.

-, « L'abolition éphémère du clivage entre intellectuels et politique durant la première coalition en Allemagne ", Revue d'Allemagne, octobre-décembre 2008.

-, « Le 20 juillet 1944 dans la presse ouest-allemande (1946-1954) ou l'édification d'un mythe fondateur ", dans M. Mathien et R. Latouche (dir.), Mémoire, histoire et médias, Bruxelles, Bruylant, 2009, p. 597-617. 
-, «L'implantation du parti Die Linke à l'Ouest de l'Allemagne: la genèse d'une redistribution des forces de gauche", Revue d'Allemagne, avril-juin 2009.

- et Hélène Camarade (dir.), Mémoires et représentations de la résistance allemande au national-socialisme en RDA et en République fédérale d'Allemagne, Revue d'Allemagne, $\mathrm{n}^{\circ} 4$, octobre-décembre 2010, $208 \mathrm{p}$.

Courriel : <nwillmann@t-online.de> 